\title{
Endocrine modifications and interventions during critical illness
}

\author{
Frank Weekers and Greet Van den Berghe* \\ Department of Intensive Care Medicine, University Hospital Leuven, Herestraat 49, 3000 Leuven, Belgium
}

\begin{abstract}
The ongoing hypermetabolic response in patients with prolonged critical illness leads to the loss of lean tissue mass. Since the cachexia of prolonged illness is usually associated with low concentrations of anabolic hormones, hormonal intervention has been thought to be beneficial. However, most interventions have been shown to be ineffective and their indiscriminate use even causes harm. Before considering endocrine intervention in these frail patients, a detailed understanding of the neuroendocrinology of the stress response is warranted. It is now clear that the acute phase and the later phase of critical illness behave differently from an endocrinological point of view. The acute stress reponse consists primarily of an activelysecreting pituitary in the presence of low circulating peripheral anabolic hormones, suggesting resistance of the peripheral tissues to the effects of anterior pituitary hormones. However, when the disease process becomes prolonged, there is a uniformly-reduced pulsatile secretion of anterior pituitary hormones with proportionally reduced concentrations of peripheral anabolic hormones. The origin of this suppressed pituitary secretion is located in the hypothalamus, as hypothalamic secretagogues can reactivate the anterior pituitary and restore pulsatile secretion. The reactivated pituitary secretion is accompanied by an increase in peripheral target hormones, indicating at least partial sensitivity of these tissues to anterior pituitary hormones in this chronic phase of illness. Thus, endocrine intervention with a combination of hypothalamic secretagogues that more completely reactivate the anterior pituitary could be a more physiological and effective strategy for inducing anabolism in patients with prolonged critical illness.
\end{abstract}

Metabolism: Endocrinology: Stress

Within hours and during the first few days following an acute severe insult patients develop clinical manifestations such as fever, tachypnea and tachycardia accompanied by hyperglycaemia, hypertriacylglycerolaemia and accelerated proteolysis. The main purpose of this hypermetabolic response is the provision of endogenous substrates to wounded tissues and reparative cells for healing at a time when exogenous energy intake is limited. When the underlying disease resolves, the stress response abates and recovery ensues.

With progress in intensive care medicine, however, patients now survive medical and surgical conditions that have previously been fatal. Recovery from such severe insults may take weeks and prolongation of the hypermetabolic response induces a profound erosion of lean body mass. These patients can lose up to $10 \%$ of their muscle mass per week (Gamrin et al. 1997) because of the ongoing proteolysis. The loss of protein is not confined to the skeletal muscle but also occurs in other organs, such as the liver, kidney and heart, reducing their cell mass and leading to impaired function. Extended recuperation often includes prolonged duration of mechanical ventilatory support because of extreme muscle weakness and fatigue. Additionally, hyperglycaemia (Liu et al. 1999) and hypertriacylglycerolaemia (Robin et al. 1989) contribute to immune dysfunction and thus increase the risk for infectious complications. Such cachectic and immunocompromised patients often die in sepsis and multiple organ failure.

As endocrine changes are invariably associated with critical illness (see Fig. 1) and in view of their role in intermediate metabolism, altered function of the anterior pituitary and its peripheral effectors may contribute to the wasting syndrome. Indeed, all critically-ill patients have

\footnotetext{
Abbreviations: GH, growth hormone; GHS, GH secretagogues; IGF-I, insulin-like growth factor I; IGFBP, IGF-binding proteins; rhGH, recombinant human $\mathrm{GH} ; \mathrm{T}_{3}$, triiodothyronine; $\mathrm{T}_{4}$, thyroxine; TSH, thyrotropin; TRH, TSH-releasing hormone.

*Corresponding author: Dr Greet Van den Berghe, fax +32 163440 15, email greta.vandenberghe@med.kuleuven.ac.be
} 




Fig. 1. A graphic representation of the dynamic changes in pituitary function during the course of critical illness. In the acute phase of illness (first hours to a few days after onset) the secretory activity of the anterior pituitary is essentially maintained or amplified, whereas anabolic target organ hormones are inactivated. Cortisol levels are elevated in concert with corticotropin. After about 7-10 d in the chronic phase of protracted critical illness the secretory activity of the anterior pituitary is uniformly suppressed, now contributing to reduced circulating levels of target organ hormones. Impaired anterior pituitary hormone secretion allows the respective target organ hormones to decrease proportionately over time, cortisol being a notable exception. Circulating levels of cortisol remain elevated through a peripheral drive, a mechanism that ultimately may also fail. The onset of recovery is characterised by restored sensitivity of the anterior pituitary to reduced feedback control. (Adapted from Van den Berghe et al. 1998.)

low serum concentrations of anabolic hormones, such as insulin-like growth factor I (IGF-I) and testosterone. As recombinant human growth hormone (rhGH) replacement has been used for many years in GH-deficient patients, increasing lean body mass in this condition (Binnerts et al. 1992), it has been thought that rhGH treatment may benefit the profoundly catabolic intensive care patient. The combination of an activated GH secretion in the presence of low peripheral IGF-I concentrations, consistently found following acute stress, has been correctly interpreted as representing a state of $\mathrm{GH}$ resistance. Additionally, anecdotal evidence has shown that rhGH has a reduced efficacy in generating IGF-I in patients with sepsis, again pointing to the presence of GH resistance (Ross et al. 1993). It has been assumed that this $\mathrm{GH}$ resistance persists throughout the course of critical illness and that high doses of rhGH would thus be required in order to overcome it and to induce anabolism. As numerous studies, mostly small and non-randomized, had reported positive effects of high-dose rhGH treatment in hypercatabolic patients, a large multicentre European trial investigating the effects of pharmacological doses of rhGH was initiated in the mid-1990s by Takala et al. (1999). Quite unexpectedly, this study revealed a marked increase in morbidity and mortality, the cause of which was unclear.

At the initiation of the Takala et al. (1999) study, the neuroendocrine changes occurring during prolonged severe illness were largely unknown. Rather, as explained earlier, data obtained from acute catabolic states were merely extrapolated to the prolonged critical illness setting, an assumption that was clearly erroneous.
It is now known that both acute and prolonged critical illness are associated with completely different neuroendocrine characteristics (Van den Berghe et al. 1998; Weekers et al. 2002). Indeed, in prolonged critical illness somatotrophs no longer actively secrete GH. Instead, a state of relative hyposomatotropism follows the initial stress response, in which pulsatile GH secretion is reduced to levels that are in proportion to the low IGF-I concentrations. In this chronic phase of illness at least partial recovery of peripheral GH sensitivity occurs. Administration of pharmacological doses of rhGH at a time of recovered GH sensitivity may expose patients to toxic side effects of such an intervention. In particular, the aggravation of insulin resistance by $\mathrm{rhGH}$ and the concomitant hyperglycaemia may have important consequences for critically-ill patients (Van den Berghe et al. 2001b). The biphasic response, first described in the $\mathrm{GH}$ axis, is also present in the thyroid, prolactin and gonadal axes, and this relative 'panhypopituitarism' could aggravate GH toxicity. In the following review the neuroendocrine changes associated with critical illness will be discussed and the current strategies to reverse hypercatabolism reappraised.

\section{Endocrine changes associated with critical illness}

The hypothalamic-anterior pituitary-peripheral hormonal axes play a central role in the endocrine regulation of the majority of metabolic processes. As even partial dysfunction of the anterior pituitary is associated with increased morbidity and mortality, there is a growing interest among intensive care specialists in the neuroendocrine 
pathophysiology of critical illness. Until recently, it has been commonly believed that the endocrine changes evoked by acute illness persist even when the disease is prolonged, although a scientific basis for this notion is lacking. In the last decade it has become clear that the anterior pituitary responds biphasically to stress and that the acute and chronic (7-10 d following injury) phases of critical illness are substantially different (Fig. 1). When studying the neuroendocrinology of critically-ill patients, a note of caution is warranted. Dopamine infusion, still one of the cornerstones in critical care medicine, profoundly suppresses the circulating concentration of all anterior pituitary-dependent hormones, except cortisol, and thus is an important confounding factor in interpreting the results from many studies (Van den Berghe \& de Zegher, 1996).

\section{Neuroendocrinology of acute illness}

GH secretion is clearly enhanced in response to acute stress. At the same time, however, the serum concentration of its main peripheral effector molecule, IGF-I, is low (Ross et al. 1991). In addition, the GH-dependent IGF-binding proteins (IGFBP) and the circulating levels of GH-binding protein, the latter presumably reflecting the functional $\mathrm{GH}$ receptor status, are low following acute stress (Hermansson et al. 1997). These findings clearly indicate the presence of GH resistance early after injury. The finding that regular doses of rhGH administered to sick patients elicit a decreased anabolic response and that there is a reduction in gene expression of the GH receptor in muscle and liver (Defalque et al. 1999) again point to the presence of $\mathrm{GH}$ resistance. Enhanced clearance of IGF-I, also partially related to changes in IGFBP, also contributes to its low serum levels. From a teleological point of view, this somatotropic response following acute injury seems appropriate. Indeed, serum concentrations of lipids and glucose are elevated by the enhanced direct lipolytic and anti-insulin effects of $\mathrm{GH}$, thus providing metabolic substrates to vital tissues, while costly anabolism, which is mediated largely by IGF-I and is considered to be less vital at this time, is postponed.

Hypercortisolism, driven by increased release of corticotropin-releasing hormone and corticotropin following illness (Rivier \& Vale, 1983), also contributes to the acute provision of energy by shifting endogenous carbohydrate, fat and protein metabolism. In addition, cortisol suppresses the endogenous inflammatory response, protecting the acutely-ill patient against over-response of this system. Furthermore, cortisol improves the haemodynamic status of the stressed individual by inducing fluid retention and by its sensitizing effect on the vasopressor response to cathecholamines.

Prolactin, presumed to be an immune-enhancing hormone, is a well-known stress hormone and the elevated serum concentrations following acute stress are thought to contribute to the activated state of the immune system early in the disease process.

While the somatotropic, adrenocortical and lactotropic axes are clearly activated in the acute phase following injury or disease, such an activation within the thyroid and gonadal axes is much more short lived. The thyroid axis responds to acute stress with a rapid drop in triiodothyronine $\left(\mathrm{T}_{3}\right)$ and an increase in reverse $\mathrm{T}_{3}$, partially related to an alternative peripheral conversion of thyroxine $\left(\mathrm{T}_{4}\right)$. Thyrotropin (TSH) concentrations are only transiently elevated and then normalize. This normalization is considered inappropriate in the face of the low $T_{4}$ and $T_{3}$ concentrations, and thus altered central feedback settings are likely (Bacci et al. 1982). As thyroid hormones are implicated in the regulation of energy expenditure and protein synthesis, their low activity in acute illness could be seen as a beneficial adaptation at times when exogenous provision of substrates is reduced.

Finally, despite elevated luteinizing hormone concentrations (Wang et al. 1978), Leydig cells are suppressed soon after injury or disease, resulting in low testosterone concentrations.

Thus, while the anterior pituitary is actively secreting in the acute phase following illness, target organs become resistant, resulting in low concentrations of their peripheral effector molecules. These endocrine and metabolic responses to acute stress are probably evolutionary adaptations and are likely to be beneficial as they reduce energy and substrate consumption, drive substrates to vital tissues, postpone anabolism and modulate the immune response in order to improve survival. Currently, there is no evidence that intervention with, or manipulation of, this response would be advantageous for the acutely-ill patient.

\section{Neuroendocrine changes in prolonged critical illness}

Progress in critical care medicine has enabled patients to survive insults that have previously been fatal. It is not likely, therefore, that nature has selected coping mechanisms for the chronic phase of the disease process. In this later phase of illness the continuing hypercatabolism leads to a non-specific wasting syndrome, including severe muscle weakness, intestinal atrophy, delayed tissue healing and immune dysfunction. The pathophysiology underlying this catabolic response in prolonged critical illness is not clear. Severe illness and tissue damage is invariably accompanied by an inflammatory response characterized by elevated levels of pro-inflammatory cytokines (TNF- $\alpha$, IL-1 and IL-6) and counter-regulatory hormones (catecholamines, glucocorticoids and glucagon) that have a profound influence on body metabolism. However, although circulating levels of these hormones are high in response to acute stress, with prolongation of the disease process serum concentrations of most of these mediators usually normalize and thus are unlikely to contribute to the wasting syndrome of prolonged critical illness.

In view of their role in intermediate metabolism and the immune system, anterior pituitary hormones may have a role in the wasting syndrome associated with prolonged critical illness.

The somatotropic axis in the chronic phase of critical illness. In contrast to the first few days following illness, when release of $\mathrm{GH}$ from somatotrophs is increased while the concentration of IGF-I is low, in the chronic phase pulsatile release of $\mathrm{GH}$ is suppressed. Concomitantly, the non-pulsatile fraction of $\mathrm{GH}$ release remains somewhat elevated (Van den Berghe et al. 1997a). 
The finding of a strong and positive correlation between circulating IGF-I, IGFBP-3 and acid-labile subunit on the one hand, and the pulsatile fraction of $\mathrm{GH}$ release on the other, indicates that the loss of pulsatile GH release in the chronic phase of critical illness probably contributes to the low IGF-I, IGFBP-3 and acid-labile subunit concentrations in this condition (Van den Berghe et al. 1999). This finding conflicts with the previous presumption of $\mathrm{GH}$ resistance, as an inverse relationship would then be expected. Moreover, the clear increase in IGF-I and GH-dependent IGFBP concentrations after infusion of GH secretagogues (GHS; Van den Berghe et al. 2000) and the presence of elevated GH-binding protein concentrations in this phase (Van den Berghe et al. 1996) indicate at least some recovery of $\mathrm{GH}$ responsiveness during prolonged severe illness.

The mechanism underlying this relative hyposomatotropism is likely to be located in the hypothalamus. Indeed, the impaired GH secretion reacts robustly to the injection of GHS, thus excluding a pituitary deficiency of GH as the mechanism underlying the relative hyposomatotropism (Van den Berghe et al. 1996, 1997a). The GH release observed following the injection of GH-releasing hormone is less pronounced than that following GHS injection. This finding suggests that, if a reduced hypothalamic drive is responsible for the hyposomatotropism, a hypothalamic deficiency of GHS is more plausible than GH-releasing hormone deficiency. Reduced somatostatin tone could also explain the profound response to GHS but cannot be reconciled with the spontaneous dynamics of lowamplitude GH bursts. This (relative) hyposomatotropism associated with prolonged critical illness is not unimportant, as it has been shown to contribute to the wasting syndrome (Van den Berghe et al. 1999). If GHS deficiency is a mechanism involved in the relative hyposomatotropism during prolonged critical illness and the recovery of peripheral GH sensitivity in this phase of illness, administration of GHS may have potential in the management of feeding-resistant hypercatabolism. Another important aspect of this treatment would be its safety. Indeed, as endogenous negative feedback mechanisms are intact in the patient with prolonged critical illness, the use of GHS would prevent over-stimulation of the somatotropic axis. It has been shown that GHS infusion results in a reactivation of pulsatile $\mathrm{GH}$ secretion and increases IGF-I concentrations up to a certain level above which further stimulation of $\mathrm{GH}$ secretion has little or no additional effects on IGF-I concentrations. In contrast, the administration of peripherally-active hormones at an inappropriate (too high) dose may expose these very sick patients to toxic side effects of such a therapy.

The thyrotropic axis in the chronic phase of critical illness. As for the somatotropic axis, circulating levels of TSH measured in a single sample are often within the low normal range, but the pulsatile fraction of TSH release is dramatically reduced during prolonged illness (Van den Berghe et al. 1998). In addition, the serum concentrations of $\mathrm{T}_{4}$ and, particularly, $\mathrm{T}_{3}$ are low and correlate positively with the reduced pulsatile TSH release (Van den Berghe et al. 1997b). The low $\mathrm{T}_{4}$ and $\mathrm{T}_{3}$ levels present when TSH release is normal or reduced suggest an altered feedback inhibition set point, impaired TSH synthesis, inadequate TSH stimulation by TSH-releasing hormone (TRH) or elevated somatostatin tone. In critically-ill patients TRH infusion increases TSH secretion and, concomitantly, peripheral thyroid hormone levels (Van den Berghe et al. 1998). As for the somatotropic axis, these data are consistent with a predominantly central origin of the suppressed thyroid axis, suggesting reduced TRH activity in the chronic phase of critical illness. The indirect evidence provided by dynamic clinical studies is supported by the work of Fliers et al. (1998), which has confirmed reduced TRH mRNA levels in the hypothalamus of patients dying after chronic critical illness compared with those who died after a road accident or an acute illness. A role of reduced GHS action in the pathophysiology of the suppressed thyroid axis has also been suggested. Indeed, pulsatile TSH release is only increased with TRH infusions when GHS are infused concomitantly (Van den Berghe et al. 1998). These findings have largely been confirmed in a rabbit model of critical illness (Weekers et al. 2002). In the acute phase of illness TRH injection induces a hyper-responsive release of TSH that wanes in the chronic phase. It has been hypothesized that GHS deficiency accounts for this finding, as the full effect of TRH apparently requires sufficient endogenous GHS availability (Van den Berghe et al. 2002).

Low- $\mathrm{T}_{3}$ syndrome results not only from alterations in the pituitary, but also from changes in peripheral metabolism. It has previously been shown that the reduced $\mathrm{T}_{3}$ levels in patients with prolonged critical illness are in part a result of reduced type 1 deiodinase activity, the enzyme responsible for the peripheral conversion of $\mathrm{T}_{4}$ to active $\mathrm{T}_{3}$ (Peeters et al. 2003). Interestingly, the addition of GH-releasing peptide-2 to TRH infusion not only stimulates pulsatile TSH release and circulating $\mathrm{T}_{4}$ and $\mathrm{T}_{3}$, but also prevents the rise in reverse $\mathrm{T}_{3}$. This finding suggests an effect of GH-releasing peptide-2 on deiodinase activity, either directly or indirectly through its effect on GH and IGF-I. In an animal model combined GHRP and TRH infusion augments the catalytic activity of hepatic type 1 deiodinase activity and depresses the activity of the main peripheral hormone inactivator enzyme, type 3 deiodinase (Weekers et al. 2004). The low- $\mathrm{T}_{3}$ syndrome in these patients is relevant, as it has been related to hypercatabolism (Van den Berghe et al. 1999).

The gonadal and prolactin axes in the chronic phase of critical illness. Hypogonadotropism occurs with prolonged critical illness. Indeed, in men with prolonged critical illness mean luteinizing hormone concentrations and pulsatile luteinizing hormone release have been shown to be suppressed even when serum testosterone concentrations are extremely low. The profound hypoandrogenism appears to be the result of a combined central and peripheral defect within the male gonadal axis (Van den Berghe et al. 2001a). Similar changes have been reported for the prolactin axis. Although prolactin acutely increases in response to stress, the pulsatile fraction of prolactin release becomes suppressed with prolongation of the disease process (Van den Berghe et al. 1997b; Weekers et al. 2002), and it has been suggested that these changes may contribute to the immune suppression associated with prolonged critical illness. 
It can be concluded that the anterior pituitary reacts biphasically to severe stress. In contrast to the acute phase of illness, prolonged critical illness is associated with a uniform suppression of the pituitary hormone axes. This process contributes to the low serum concentrations of the respective target organ hormones. Evidence now points to a predominantly hypothalamic origin of these alterations.

\section{Interventions to attenuate the catabolic syndrome of prolonged critical illness}

As hypercatabolism has severe consequences for the critically-ill patient, the quest for strategies to efficiently reverse the loss of lean body mass has been ongoing for some time.

Parenteral nutrition has been advocated as an effective intervention to halt protein loss and muscle wasting. Early studies have shown a beneficial effect of both glucose and amino acid infusions on post-operative protein sparing, leading to the provision of parenteral nutrition in the post-operative setting. Critically-ill patients, however, are frequently underfed and just increasing energy intake or protein supply is now known to be insufficient to prevent or reverse muscle wasting in hypercatabolic patients. In fact, this strategy merely results in fat accretion (Streat et al. 1987) and enhanced, instead of reduced, $\mathrm{N}$ loss. In addition, a recent meta-analysis has concluded that parenteral nutrition is not entirely harmless and may be associated with a higher incidence of complications, particularly in critically-ill patients (Heyland et al. 1998). Thus, new nutritional formulations have been studied. A high-carbohydrate enteral feeding regimen has been shown to decrease muscle protein breakdown in severelyburned children, probably by stimulating insulin release (Hart et al. 2001). However, a glucose-rich parenteral feeding regimen in critically-ill patients has been shown to induce hyperglycaemia, to increase $\mathrm{CO}_{2}$ production and to stimulate de novo lipogenesis. Furthermore, it does not suppress gluconeogenesis and proteolysis. The development of hyperglycaemia is particularly problematic in view of the important effects of stress hyperglycaemia on morbidity and mortality in critically-ill patients (Van den Berghe et al. 2001b). Several small studies have shown that parenteral and enteral feeding formulations enriched with specific supplements, such as glutamine and arginine, may be beneficial for certain patients, although a recent meta-analysis has also suggested potentially harmful effects (Heyland et al. 1998). Thus, it is necessary to await confirmation from large clinical trials before these new nutritional formulations enter widespread clinical use.

As a consequence of the failures encountered with nutritional interventions during the last decade, pharmacological enhancement of energy support has been evaluated. The non-selective $\beta$-blocker, propanolol, has a marked effect on the preservation of lean body mass in severelyburned children, mainly through a more efficient endogenous use of amino acids, which increases protein synthesis (Herndon et al. 2001).

Insulin has clear anabolic actions in patients with burn injuries, and can improve net protein balance by increasing protein synthesis or reducing protein degradation. Both effects may be related to a more efficient incorporation of amino acids into muscle. While the effect of insulin on proteolysis has been shown consistently, only extreme hyperinsulinaemia has been demonstrated to stimulate protein synthesis (Hillier et al. 1998). Liver biopsies from patients with prolonged critical illness show that insulin treatment to maintain normoglycaemia does not affect expression of phosphoenolpyruvate carboxykinase, the key enzyme controlling gluconeogenesis. In addition, in order for insulin to induce anabolism it is of paramount importance that sufficient exogenous amino acids are available, as without these amino acids insulin-stimulated protein synthesis uses amino acids derived from accelerated endogenous proteolysis. Also, it remains to be determined whether the anabolic effect of insulin is direct or mediated via stimulated IGF-I synthesis.

As with insulin, infusion of IGF-I has been shown to inhibit protein breakdown and stimulate protein synthesis in the presence of sufficient amino acids (Russell-Jones et al. 1994) and improved post-operative catabolism. Prolonged administration, however, results in reduced efficacy.

As several anterior pituitary-dependent hormones are extensively involved in the regulation of intermediate metabolism, and in view of the relative hypopituitarism associated with prolonged critical illness, it is rational to search for a role for these hormones in the treatment of catabolism. Thyroid hormones are important for protein synthesis, lipolysis and fuel utilization by muscle, and they interfere with GH secretion and tissue responsiveness to GH. At present, however, it is unclear whether treatment with thyroid hormones is beneficial or harmful for the critically-ill patient (Stathatos et al. 2001). Administration of $T_{3}$ during starvation has been shown to increase muscle wasting, and increased mortality has been observed in animal models of sepsis (Gardner et al. 1979).

Administration of testosterone to men with severe burn injuries is accompanied by a marked reduction in protein catabolism, mainly through efficient intracellular amino acid handling and inhibition of muscle breakdown (Ferrando et al. 2001). In a small study (Demling, 1999) the synthetic androgen, oxandrolone, has been shown to reduce weight loss, improve functional status and increase wound healing, and seems to be as effective as rhGH therapy in the acute and recovery phase of illness; however, caution is warranted, as these studies were not well controlled and did not report the potential complications of this intervention. Moreover, testosterone supplementation during illness may be associated with negative effects on the immune system (Angele et al. 1998).

The beneficial metabolic effects of rhGH in GH-deficient patients have been known for many years (Binnerts et al. 1992). In these patients treatment with rhGH increases lean body mass and stimulates bone remodelling. The direct effects of $\mathrm{GH}$ are diverse. It induces insulin insensitivity (Costa et al. 1998) and stimulates lipolysis (Keller \& Miles, 1991), inhibits the peripheral conversion of cortisone to cortisol (Stewart et al. 2001), regulates the peripheral conversion of thyroid hormones (Moller et al. 
1992) and interferes with drug metabolism. GH has also been shown to improve heart function in patients with heart failure, although it remains to be seen whether this benefit is also applicable to patients in the intensive care. In addition, GH is an immune-modulating agent and enhances innate immunity, properties that might be beneficial in the immune-deficient critically-ill patient.

Several small and often poorly-controlled studies have suggested beneficial effects of rhGH in catabolic patients, but very few of these studies have reported the potential complications. Most of these studies used relatively large doses of rhGH, as $\mathrm{GH}$ has been shown to diminish metabolic efficacy in patients with sepsis, as a result of GH resistance (Dahn et al. 1988). In elective colo-rectal surgery a $9 \mathrm{~d}$ peri-operative rhGH injection (4 and $8 \mathrm{U}$ $(1.3$ and $2.6 \mathrm{mg}) / \mathrm{d})$ has been found to improve $\mathrm{N}$ balance and lean body mass, even when patients have a low energy intake, but the treatment is associated with an average retention of 4 litres fluid (Vara-Thorbeck et al. 1992). In post-operative cancer patients whole-body net protein balance is improved following $5 \mathrm{~d}$ of $\mathrm{rhGH}$ treatment, while there is no effect on muscle protein balance unless insulin is added (Berman et al. 1999). In patients with sepsis $7 \mathrm{~d}$ of rhGH treatment improves net protein synthesis (Koea et al. 1996). rhGH treatment for $5 \mathrm{~d}$ has been shown to stimulate muscle protein synthesis and to increase glutamine stores in the muscle of patients with prolonged critical illness (Gamrin et al. 2000). In children with burns rhGH treatment is associated with faster healing of the donor site and improved protein synthesis (Gilpin et al. 1994). Furthermore, there is a reduced albumin requirement, an anti-inflammatory response (reductions in C-reactive protein, TNF $\alpha$ and IL-6) and only a transient increase in NEFA. Mjaaland et al. (1993) have found that rhGH alters substrate utilization with increased fat oxidation, fatty acids and energy expenditure and decreased carbohydrate oxidation.

The first prospective double-blind randomized controlled trial of rhGH administration in a large population of long-stay intensive care patients that was of sufficient power to analyse morbidity and mortality was undertaken by Takala et al. (1999). Prolonged critically-ill and mechanically-ventilated patients without sepsis were randomized to receive pharmacological doses of rhGH or placebo for a maximum of 3 weeks. Surprisingly, in the rhGH-treated group an excess of intensive care mortality was reported, which persisted when analysing in-hospital mortality. The main causes of death in patients receiving rhGH treatment were reported to be sepsis and multiple organ failure. Furthermore, surviving patients had longer periods of mechanical ventilation, hospitalization and higher health care costs despite increased levels of IGF-I and a positive $\mathrm{N}$ balance. Hyperglycaemia was found to be present more frequently in the rhGH-treated patients compared with those receiving placebo. The aetiology of the dramatic effect of rhGH treatment in the criticallyill patient is not clear. It has been suggested that GH treatment may have limited glutamine release from skeletal muscle, thus reducing the supply of this amino acid for immune and gut cells. Impaired immune function and increased intestinal permeability could explain the high incidence of infections in patients treated with high-dose rhGH. This hypothesis has been questioned by findings in rats with sepsis treated with rhGH (O'Leary et al. 2002). Furthermore, GH and IGF-I have no influence on glutamine metabolism. Additionally, as glutamine is involved in the preservation of reserves of the antioxidant glutathione in the liver, the finding that early rhGH treatment in rats has an antioxidant effect, with reduced lipid peroxidation in lung and liver, and increased tissue levels of glutathione, does not support such a hypothesis (Youn et al. 1998).

Liao et al. (1996) have reported that rhGH pretreatment (by continuous infusion) followed by endotoxin injection in rats results in a worse outcome, with profound shock, hypertriacylglycerolaemia and liver and kidney failure. This effect is independent of IGF-I and is presumed to be related to the priming of lymphocytes. In agreement with this notion are reports of a worsening of acidosis associated with reduced portal vein flow and higher TNF- $\alpha$ levels in an animal model of sepsis that was treated with rhGH (Unneberg et al. 1996).

In view of recent data on the neuroendocrine pathophysiology of prolonged critical illness, the findings of the study by Takala et al. (1999) can be interpreted from another perspective. It is now clear that $\mathrm{GH}$ secretion is suppressed and GH responsiveness recovers in the prolonged phase of critical illness. Hence, the high dose of rhGH used in the multicentre trial was probably much too high, exposing these patients to the potentially deleterious direct effects of GH. Furthermore, the finding that multiple endocrine axes are inactivated in critical illness suggests that endocrine intervention should target the somatotropic, thyrotropic and gonadal axes.

It has been shown (Van den Berghe et al. 1999) that infusion of GH-releasing peptide-2, together with TRH, for $5 \mathrm{~d}$ reactivates both the somatotropic and thyrotropic axes in patients with prolonged critical illness. Basal and pulsatile GH release and circulating levels of IGF-I, IGFBP-3, acid-labile subunit and IGFBP-5 are all stimulated. Concomitantly, the secretion of TSH and $\mathrm{T}_{4}$ and $\mathrm{T}_{3}$ release are amplified without affecting levels of reverse $\mathrm{T}_{3}$. Feedback inhibition loops remain active, thus preventing overstimulation. IGF-I increases to normal levels during the first $48 \mathrm{~h}$ of treatment and then stabilizes. The clear reactivation of the $\mathrm{GH}$ and thyroid axes that result from the administration of these hypothalamic releasing factors is associated after $5 \mathrm{~d}$ with a reduction in hypercatabolism and the stimulation of anabolism. The rate of proteolysis is markedly reduced and this effect is predominantly related to the correction of the tertiary hypothyroidism. Osteocalcin, a surrogate marker for anabolism, is increased with this treatment, as a result of improved maturation of osteoblasts, and this anabolic effect could be explained largely by the rise in IGF-I and GH-dependent IGFBP. Recently, it has been found that co-activation of the male gonadal axis using gonadotropin-releasing hormone, in addition to the infusion of GH-releasing peptide-2 and TRH, i.e. restoration of the three pituitary axes, in critically-ill patients is associated with an even more pronounced anabolic effect (Van den Berghe et al. 2001a). 


\section{Conclusion}

Patients with critical illness face a dramatic loss of lean body mass when their disease process becomes prolonged. Routine intensive care interventions, such as feeding, are unable to reverse this catabolic state and they delay recuperation of these patients. Changes in anterior pituitary function are invariably associated with critical illness. The acute phase is characterized by an actively-secreting pituitary, whereas the peripheral tissues are resistant to the effects of anterior pituitary hormones, resulting in low concentrations of their effector molecules. It was originally assumed that this condition persists with ongoing disease. As a result, interventions with high doses of single hormone treatment, such as rhGH, have been deemed to be necessary in order to induce anabolism. The results of these interventions have been disappointing and some of them have caused severe harm.

With prolonged illness, it is now know that there is a reduced secretion of hormones by the anterior pituitary, which causes reduced concentrations of peripheral effector hormones and contributes to the general wasting syndrome in these patients. Importantly, infusion of hypothalamicreleasing substances can reactivate the anterior pituitary, leading to an increase in peripheral effector hormones, such as IGF-I and $\mathrm{T}_{3}$. These substances, therefore, hold promise for the future, as short-term reactivation of the somatotropic, thyrotropic and gonadal axes has been shown to be associated with improved anabolism and attenuated catabolism. Large-scale clinical studies examining the effect of hypothalamic secretagogues on the outcome of critical illness should now be initiated.

\section{References}

Angele MK, Ayala A, Cioffi WG, Bland KI \& Chaudry IH (1998) Testosterone: the culprit for producing splenocyte immune depression after trauma hemorrhage. American Journal of Physiology 274, C1530-C1536.

Bacci V, Schussler GC \& Kaplan TB (1982) The relationship between serum triiodothyronine and thyrotropin during systemic illness. Journal of Clinical Endocrinology and Metabolism 54, 1229-1235.

Berman R, Harrison L, Pearlstone D, Burst M \& Brennan M (1999) Growth hormone, alone and in combination with insulin, increases whole body and skeletal muscle protein kinetics in cancer patients after surgery. Annals of Surgery 229, $1-10$.

Binnerts A, Swart G, Wilson J, Hoogerbrugge N, Pols H, Birkenhager J \& Lamberts S (1992) The effects of growth hormone administration in growth hormone deficient adults on bone, protein, carbohydrate and lipid homeostasis, as well as on body composition. Clinical Endocrinology 37, 79-87.

Costa C, Solanes G, Visa J \& Bosch F (1998) Transgenic rabbits overexpressing growth hormone develop acromegaly and diabetes mellitus. FASEB Journal 12, 1455-1460.

Dahn MS, Lange MP \& Jacobs LA (1988) Insulin-like growth factor 1 production is inhibited in human sepsis. Archives of Surgery 123, 1409-1414.

Defalque D, Brandt N, Ketelslegers J \& Thissen J (1999) GH insensitivity induced by endotoxin injection is associated with decreased liver GH receptors. American Journal of Physiology 276, E565-E572.
Demling RH (1999) Comparison of the anabolic effects and complications of human growth hormone and the testosterone analog, oxandrolone, after severe burn injury. Burns $\mathbf{2 5}$, 215-221.

Ferrando AA, Sheffield-Moore M, Wolf SE, Herndon DN \& Wolfe RR (2001) Testosterone administration in severe burns ameliorates muscle catabolism. Critical Care Medicine 10 1936-1942.

Fliers E, Wiersinga WM \& Swaab DF (1998) Physiological and pathophysiological aspects of thyrotropin-releasing hormone gene expression in the human hypothalamus. Thyroid $\mathbf{8}$, 921-928.

Gamrin L, Andersson K, Hultman E, Nilsson E, Essen P \& Wernerman J (1997) Longitudinal changes of biochemical parameters in muscle during critical illness. Metabolism 46, 756-762.

Gamrin L, Essen P, Hultman E, McNurlan M, Garlick P \& Wernerman J (2000) Protein sparing effect in skeletal muscle of growth hormone treatment in critically ill patients. Annals of Surgery 231, 577-586.

Gardner DF, Kaplan MM, Stanley CA \& Utiger RD (1979) Effect of tri-iodothyronine replacement on the metabolic and pituitary responses to starvation. New England Journal of Medicine 300, 579-584.

Gilpin DA, Barrow RE, Rutan RL, Broemeling L \& Herndon DN (1994) Recombinant human growth hormone accelerates wound healing in children with large cutaneous burns. Annals of Surgery 220, 19-24.

Hart DW, Wolf SE, Zhang XJ, Chinkes DL, Buffalo MC, Matin SI, DebRoy MA, Wolfe RR \& Herndon DN (2001) Efficacy of a high-carbohydrate diet in catabolic illness. Critical Care Medicine 29, 1318-1324.

Hermansson M, Wickelgren RB, Hammarqvist F, Bjarnason R, Wennstrom I, Wernerman J, Carlsson B \& Carlsson LM (1997) Measurement of human growth hormone receptor messenger ribonucleic acid by a quantitative polymerase chain reactionbased assay: demonstration of reduced expression after elective surgery. Journal of Clinical Endocrinology and Metabolism 82, 421-428.

Herndon DN, Hart DW, Wolf SE, Chinkes DL \& Wolfe RR (2001) Reversal of catabolism by beta-blockade after severe burns. New England Journal of Medicine 345, 122-129.

Heyland DK, MacDonald S, Keefe L \& Drover JW (1998) Total parenteral nutrition in the critically ill patient: a meta-analysis. Journal of the American Medical Association 280, 2013-2019.

Hillier TA, Fryburg DA, Jahn LA \& Barrett EJ (1998) Extreme hyperinsulinemia unmasks insulin's effect to stimulate protein synthesis in the human forearm. American Journal of Physiology 274, E1067-E1074.

Keller U \& Miles J (1991) Growth hormone and lipids. Hormone Research 36, 36-40.

Koea J, Breier B, Douglas R, Gluckman P \& Shaw J (1996) Anabolic and cardiovascular effects of recombinant human growth hormone in surgical patients with sepsis. British Journal of Surgery 83, 196-202.

Liao W, Rudling M \& Angelin B (1996) Growth hormone potentiates the in vivo biological activities of endotoxin in the rat. European Journal of Clinical Investigation 26, 254-258.

Liu B, Miyata S, Kojima A, Kusunoki H, Suzuki K \& Kasuga M (1999) Low phagocytic activity of resident peritoneal macrophages in diabetic mice: relevance to the formation of advanced glycation end procucts. Diabetes 48, 2074-2082.

Mjaaland M, Unneberg K, Larsson J, Nilsson L \& Revhaug A (1993) Growth hormone after abdominal surgery attenuated forearm glutamine, alanine, 3-methylhistidine, and total amino acid efflux in patients receiving total parenteral nutrition. Annals of Surgery 217, 413-422. 
Moller J, Jorgensen J, Moller N, Christiansen J \& Weeke J (1992) Effects of growth hormone administration on fuel oxidation and thyroid function in normal man. Metabolism 41, 728-731.

O'Leary M, Fergusson C, Rennie M, Hinds C, Coakley J \& Preedy V (2002) Effect of growth hormone on muscle and liver protein synthesis in septic rats receiving glutamine-enriched parenteral nutrition. Critical Care Medicine 30, 1099-1105.

Peeters RP, Wouters PJ, Kaptein E, van Toor H, Visser TJ \& Van den Berghe G (2003) Reduced activation and increased inactivation of thyroid hormone in tissues of critically ill patients. Journal of Clinical Endocrinology and Metabolism 88, 3202-3211.

Rivier C \& Vale W (1983) Modulation of stress-induced ACTH release by corticotropin-releasing factor, catecholamines and vasopressin. Nature 305, 325-327.

Robin P, Arain I, Phuangsab A, Holian O, Roccaforte P \& Barrett JA (1989) Intravenous fat emulsion acutely suppresses neutrophil chemiluminescence. Journal of Parenteral and Enteral Nutrition 13, 608-613.

Ross R, Miell J, Freeman E, Jones J, Matthews D, Preece M \& Buchanan C (1991) Critically ill patients have high basal growth hormone levels with attenuated oscillatory activity associated with low levels of insulin-like growth factor-I. Clinical Endocrinology 35, 47-54.

Ross RJ, Rodriguez-Arnao J, Bentham J \& Coakley JH (1993) The role of insulin, growth hormone and IGF-I as anabolic agents in the critically ill. Intensive Care Medicine 19, S54-S57.

Russell-Jones DL, Umpleby AM, Hennessy TR, Bowes SB, Shojaee-Moradie F, Hopkins KD, Jackson NC, Kelly JM, Jones RH \& Sonksen P (1994) Use of a leucine clamp to demonstrate that IGF-I actively stimulates protein synthesis in normal humans. American Journal of Physiology 267, E591-E598.

Stathatos N, Levetan C, Burman KD \& Wartofsky L (2001) The controversy of the treatment of critically ill patients with thyroid hormone. Best Practise Research in Clinical Endocrinology and Metabolism 15, 465-478.

Stewart P, Toogood A \& Tomlinson J (2001) Growth hormone, insulin-like growth factor-I and the cortisol-cortisone shuttle. Hormone Research 56, 1-6.

Streat SJ, Beddoe AH \& Hill GL (1987) Aggressive nutritional support does not prevent protein loss despite fat gain in septic intensive care patients. Journal of Trauma 27, 262-266.

Takala J, Ruokonen E, Webster NR, Nielsen MS, Zandstra DF, Vundelinckx G \& Hinds CJ (1999) Increased mortality associated with growth hormone treatment in critically ill adults. New England Journal of Medicine 341, 785-792.

Unneberg K, Balteskard L, Mjaaland M \& Revhaug A (1996) Growth hormone impaired compensation of hemorrhagic shock after trauma and sepsis in swine. Journal of Trauma 41, 775-780.

Van den Berghe G, Baxter RC, Weekers F, Wouters P, Bowers CY, Iranmanesh A, Veldhuis JD \& Bouillon R (2002) The combined administration of GH-releasing peptide-2 (GHRP-2), TRH and GnRH to men with prolonged critical illness evokes superior endocrine and metabolic effects compared to treatment with GHRP-2 alone. Clinical Endocrinology 56, 655-669.

Van den Berghe G, Baxter RC, Weekers F, Wouters P, Bowers CY \& Veldhuis JD (2000) A paradoxical gender dissociation within the growth hormone/insulin-like growth factor I axis during protracted critical illness. Journal of Clinical Endocrinology and Metabolism 85, 183-192.

Van den Berghe G \& de Zegher F (1996) Anterior pituitary function during critical illness and dopamine treatment. Critical Care Medicine 24, 1580-1590.
Van den Berghe G, de Zegher F \& Bouillon R (1998) Acute and prolonged critical illness as different neuroendocrine paradigms. Journal of Clinical Endocrinology and Metabolism 83, $1827-1834$.

Van den Berghe G, de Zegher F, Bowers CY, Wouters P, Muller P, Soetens F, Vlasselaers D, Schetz M, Verwaest C, Lauwers P \& Bouillon R (1996) Pituitary responsiveness to GH-releasing hormone, GH-releasing peptide-2 and thyrotrophin-releasing hormone in critical illness. Clinical Endocrinology $\mathbf{4 5}$, 341-351.

Van den Berghe G, de Zegher F, Veldhuis JD, Wouters P, Awouters M, Verbruggen W, Schetz M, Verwaest C, Lauwers P, Bouillon R \& Bowers CY (1997a). The somatotropic axis in critical illness: effect of continuous growth hormone (GH)-releasing hormone and GH-releasing peptide-2 infusion. Journal of Clinical Endocrinology and Metabolism 82, 590-599.

Van den Berghe G, de Zegher F, Veldhuis JD, Wouters P, Gouwy S, Stockman W, Weekers F, Schetz M, Lauwers P, Bouillon R \& Bowers CY (1997b) Thyrotrophin and prolactin release in prolonged critical illness: dynamics of spontaneous secretion and effects of growth hormone-secretagogues. Clinical Endocrinology 47, 599-612.

Van den Berghe $G$, Weekers F, Baxter RC, Wouters P, Iranmanesh A, Bouillon R \& Veldhuis JD (2001a) Five-day pulsatile gonadotropin-releasing hormone administration unveils combined hypothalamic-pituitary-gonadal defects underlying profound hypoandrogenism in men with prolonged critical illness. Journal of Clinical Endocrinology and Metabolism 86, 3217-3226.

Van den Berghe G, Wouters P, Weekers F, Mohan S, Baxter RC, Veldhuis JD, Bowers CY \& Bouillon R (1999) Reactivation of pituitary hormone release and metabolic improvement by infusion of growth hormone-releasing peptide and thyrotropinreleasing hormone in patients with protracted critical illness. Journal of Clinical Endocrinology and Metabolism 84, 1311-1323.

Van den Berghe G, Wouters P, Weekers F, Verwaest C, Bruyninckx F, Schetz M, Vlasselaers D, Ferdinande P, Lauwers P \& Bouillon R (2001b) Intensive insulin therapy in the critically ill patients. New England Journal of Medicine 345, 1359-1367.

Vara-Thorbeck R, Guerrero JA, Ruiz-Requena ME, Capitan J, Rodriguez M, Rosell J, Mekinassi K, Maldonado M \& Martin $\mathrm{R}$ (1992) Effects of growth hormone in patients receiving total parenteral nutrition following major gastrointestinal surgery. Hepatogastroenterology 39, 270-272.

Wang C, Chan V \& Yeung RT (1978) Effect of surgical stress on pituitary-testicular function. Clinical Endocrinology 9, 255-266.

Weekers F, Michalaki M, Coopmans W, Van Herck E, Veldhuis JD, Darras VM \& Van den Berghe G (2004) Endocrine and metabolic effects of growth hormone $(\mathrm{GH})$ compared with GH-releasing peptide, thyrotropin-releasing hormone and insulin infusion in a rabbit model of prolonged critical illness. Endocrinology 145, 205-213.

Weekers F, Van Herck E, Coopmans W, Michalaki M, Bowers CY, Veldhuis JD \& Van den Berghe G (2002) A novel in vivo rabbit model of hypercatabolic critical illness reveals a biphasic neuroendocrine stress response. Endocrinology 143, 764-774.

Youn Y, Suh G, Jung S, Oh S \& Demling R (1998) Recombinant human growth hormone decreases lung and liver tissue lipid peroxidation and increases antioxidant activity after thermal injury. Journal of Burn Care and Rehabilitation 19, 542-548. 